\title{
Editorial: Developments in Campylobacter, Helicobacter \& Related Organisms Research - CHRO 2019
}

\author{
Nicolae Corcionivoschi ${ }^{1}$, Stuart A. Thompson ${ }^{2}$ and Ozan Gundogdu ${ }^{3 *}$ \\ ${ }^{1}$ Bacteriology Branch, Veterinary Sciences Division, Agri-Food and Biosciences Institute (AFBI), Belfast, United Kingdom, \\ ${ }^{2}$ Medical College of Georgia, Augusta University, Augusta, GA, United States, ${ }^{3}$ Department of Infection Biology, Faculty of \\ Infectious and Tropical Diseases, London School of Hygiene and Tropical Medicine, London, United Kingdom
}

Keywords: Campylobacter, Helicobacter, CHRO 2019, gastrointestinal pathogen, Campylobacter Helicobacter and Related Organisms

\section{Editorial on the Research Topic}

\section{Developments in Campylobacter, Helicobacter \& Related Organisms Research - CHRO 2019}

Campylobacter spp. and Helicobacter spp. are important gastrointestinal pathogens that are major causes of acute gastroenteritis and gastric disease, respectively (Polk and Peek, 2010; Gundogdu and Wren, 2020). Campylobacter spp. are considered the leading bacterial cause of human gastroenteritis. In low-resource settings, Campylobacter infections are common in young children and correlate with stunted growth and life-long physical and cognitive deficiencies (Amour et al., 2016). In high-resource regions, an estimated 1 in every 100 individuals develop a Campylobacter-related illness each year. Helicobacter spp. can colonize the human stomach and increase the risk of ulcers and stomach cancer (Salama et al., 2013). Helicobacter pylori is the most common species with some reports indicating up to $50 \%$ of the population are infected (Brown, 2000). Both Campylobacter spp. and Helicobacter spp. possess a plethora of survival and virulence factors that have allowed them to survive and persist successfully (Gundogdu et al., 2016; Hathroubi et al., 2018; Capurro et al., 2019; El Abbar et al., 2019; Liaw et al., 2019). For both Campylobacter spp. and Helicobacter spp., contaminated foods play an important role in the transmission of the microorganism to humans (Tegtmeyer et al., 2017; Ijaz et al., 2018; Quaglia and Dambrosio, 2018; Sibanda et al., 2018; McKenna et al., 2020).

The 20th International workshop on Campylobacter, Helicobacter, and Related Organisms (CHRO) was held in Belfast, Northern Ireland from September 8-11th, 2019. This biennial conference provided researchers with an opportunity to display the most recent findings in our understanding of Campylobacter, Helicobacter, and related organisms. The conference showcased the research from different topics ranging from pathogenicity and virulence factors; poultry and non-poultry epidemiology and ecology; emerging and related species; control strategies; outbreak/epidemiology and public health; detection methods and characterization; antibiotics and antimicrobials; bioinformatics, and genomics and evolution; immunology and host response. This Frontiers Research Topic provides a framework to showcase a selection of this current research.

A number of manuscripts focussed on the association between Campylobacter and poultry, indicating the growing importance of this research field. Quyen et al. described an optimized Loop Mediated Isothermal Amplification (LAMP) method for rapid detection of Campylobacter spp. in broilers, with increased specificity and sensitivity. Lafontaine et al. investigated the prebiotic galacto-oligosaccharide (GOS) on broiler chickens colonized with C. jejuni. The authors identified 
that GOS-fed birds had increased growth performance, however an increased IL-17A did not prevent colonization with $C$. jejuni. Chinivasagam et al. investigated the use of bacteriophages to control Campylobacter in commercially farmed broiler chickens in Australia. Muhandiramlage et al. investigated the physiological and morphological changes on Campylobacter isolates from chicken meat that were induced with chlorine.

Studies also continued to investigate the genomics and epidemiology of strains from different sources around the globe. Abd El-Hamid et al. described the genetic diversity of C. jejuni strains isolated from avian and human sources from Egypt. Terefe et al. investigated the co-occurrence of Campylobacter spp. in children from eastern Ethiopia and their association with environmental enteric dysfunction, diarrhea, and host microbiome. The authors highlighted the association between specific microbiome composition and gut permeability, gut inflammation, enteric dysfunction severity, and diarrhea. Mutschall et al. investigated C. jejuni strain dynamics in a raccoon population in southern Ontario, Canada. The authors noted that due to a high prevalence and rapid subtype turnover, racoons may act as vectors in the transmission of clinically relevant $C$. jejuni subtypes at the interface of rural, urban, and more natural environments. Hetman et al. described recovery bias of common C. jejuni subtypes in mixed cultures. The authors emphasized the importance of selecting multiple colonies per sample, using both enrichment and non-enrichment isolation procedures to maximize the probability of recovering multiple subtypes present in a sample. Phung et al. discussed the routes of infection of C. hepaticus which causes spotty liver disease in chickens. The authors highlighted that environmental sources are a likely transmission source of $C$. hepaticus.

In relation to survival, Riedel et al. analyzed the transcriptomic differences in C. jejuni and C. coli when exposed to elevated temperatures of $46^{\circ} \mathrm{C}$, identifying several chaperones with increased gene expression indicative of a general involvement within heat stress response. Lv et al. described methods to detect and quantify C. jejuni from the viable but non-culturable (VBNC) state. The authors discuss the use of PMA-qPCR as a rapid, specific and sensitive method for the detection and quantification of VBNC C. jejuni.

Research focussing on immunology and host response was presented by Butkevych et al. who discussed the impact of

\section{REFERENCES}

Amour, C., Gratz, J., Mduma, E., Svensen, E., Rogawski, E. T., Mcgrath, M., et al. (2016). Epidemiology and impact of Campylobacter infection in children in 8 low-resource settings: results from the MAL-ED study. Clin. Infect. Dis. 63, 1171-1179. doi: 10.1093/cid/ciw542

Brown, L. M. (2000). Helicobacter pylori: epidemiology and routes of transmission. Epidemiol. Rev. 22, 283-297. doi: 10.1093/oxfordjournals.epirev.a0 18040

Capurro, M. I., Greenfield, L. K., Prashar, A., Xia, S., Abdullah, M., Wong, H., et al. (2019). VacA generates a protective intracellular reservoir for Helicobacter pylori that is eliminated by activation of the lysosomal calcium channel TRPML1. Nat. Microbiol. 4, 1411-1423. doi: 10.1038/s41564-0190441-6 epithelial apoptosis and subepithelial immune responses in C. jejuni-induced barrier disruption. The authors highlighted that $C$. jejuni infection and the consequent subepithelial immune activation leads to intestinal barrier dysfunction predominantly through caspase-3-dependent epithelial apoptosis. Pathogenicity and virulence factors were investigated by $\mathrm{Li}$ et al. who describe a putative novel role for FlhF in terms of directly regulating flagellar genes and further our understanding of FlhF in relation to Campylobacter flagellar biosynthesis and flagellation. Konkel et al. provided a comprehensive review of Campylobacter adherence and invasion, specifically focussing on fibronectin and binding from CadF and FlpA adhesins. Guérin et al. investigated the membrane proteocomplexome of C. jejuni using 2-D blue native/SDS-PAGE in conjunction with bioinformatic analysis. The authors identified a range of membrane protein complexes (MCPs) in C. jejuni 81-176 where these MCPs are involved in protein folding, molecules trafficking, oxidative phosphorylation, membrane structuration, peptidoglycan biosynthesis, motility and chemotaxis, stress signaling, efflux pumps, and virulence. Duma et al. discussed the influence of protein glycosylation on C. fetus physiology. The authors used label-free quantitative (LFQ) proteomics, identifying more than 100 proteins significantly altered in expression in two C. fetus subsp. fetus protein glycosylation ( $p g l$ ) mutants ( $p g l X$ and $p g l J)$ compared to the wild-type strain. The authors provided a study which gives insight into the unique protein N-glycosylation pathway of C. fetus, but also expands our knowledge on the influence of protein $\mathrm{N}$-glycosylation on Campylobacter cell physiology. Tejera et al. performed a genome-scale metabolic model driven design of a medium for C. jejuni M1cam strain. The authors showed that with a wellcurated metabolic model, it is possible to design media to grow Campylobacter and that this has implications for the study of new Campylobacter species defined through metagenomics.

This Research Topic will increase the knowledge base and understanding of the processes of survival of Campylobacter spp. and Helicobacter spp. within the environment, in particular, relating to food safety, and to host-pathogen interactions.

\section{AUTHOR CONTRIBUTIONS}

All authors contributed to the drafting of the editorial.
El Abbar, F. M., Li, J., Owen, H. C., Daugherty, C. L., Fulmer, C. A., Bogacz, M., et al. (2019). RNA Binding by the Campylobacter jejuni post-transcriptional regulator CsrA. Front. Microbiol. 10:1776. doi: 10.3389/fmicb.2019.01776

Gundogdu, O., Da Silva, D. T., Mohammad, B., Elmi, A., Wren, B. W., Van Vliet, A. H., et al. (2016). The Campylobacter jejuni oxidative stress regulator RrpB is associated with a genomic hypervariable region and altered oxidative stress resistance. Front. Microbiol. 7:2117. doi: 10.3389/fmicb.2016.02117

Gundogdu, O., and Wren, B. W. (2020). Microbe profile: Campylobacter jejunisurvival instincts. Microbiology 166, 230-232. doi: 10.1099/mic.0.000906

Hathroubi, S., Zerebinski, J., and Ottemann, K. M. (2018). Helicobacter pylori biofilm involves a multigene stress-biased response, including a structural role for flagella. $m$ Bio 9:e01973-18. doi: 10.1128/mBio.01973-18

Ijaz, U. Z., Sivaloganathan, L., McKenna, A., Richmond, A., Kelly, C., Linton, M., et al. (2018). Comprehensive longitudinal microbiome analysis of the 
chicken cecum reveals a shift from competitive to environmental drivers and a window of opportunity for Campylobacter. Front. Microbiol. 9:2452. doi: $10.3389 /$ fmicb. 2018.02452

Liaw, J., Hong, G., Davies, C., Elmi, A., Sima, F., Stratakos, A., et al. (2019). The Campylobacter jejuni type VI secretion system enhances the oxidative stress response and host colonization. Front. Microbiol. 10:2864. doi: $10.3389 /$ fmicb. 2019.02864

McKenna, A., Ijaz, U. Z., Kelly, C., Linton, M., Sloan, W. T., Green, B. D., et al. (2020). Impact of industrial production system parameters on chicken microbiomes: mechanisms to improve performance and reduce Campylobacter. Microbiome 8:128. doi: 10.1186/s40168-020-00908-8

Polk, D. B., and Peek, R. M. Jr. (2010). Helicobacter pylori: gastric cancer and beyond. Nat. Rev. Cancer 10, 403-414. doi: 10.1038/ nrc2857

Quaglia, N. C., and Dambrosio, A. (2018). Helicobacter pylori: a foodborne pathogen? World J. Gastroenterol. 24, 3472-3487. doi: 10.3748/wjg.v24.i 31.3472

Salama, N. R., Hartung, M. L., and Muller, A. (2013). Life in the human stomach: persistence strategies of the bacterial pathogen Helicobacter pylori. Nat. Rev. Microbiol. 11, 385-399. doi: 10.1038/nrmicro3016
Sibanda, N., McKenna, A., Richmond, A., Ricke, S. C., Callaway, T., Stratakos, A. C., et al. (2018). A review of the effect of management practices on Campylobacter prevalence in poultry farms. Front. Microbiol. 9:2002. doi: 10.3389/fmicb.2018.02002

Tegtmeyer, N., Wessler, S., Necchi, V., Rohde, M., Harrer, A., Rau, T. T., et al. (2017). Helicobacter pylori employs a unique basolateral type IV secretion mechanism for CagA delivery. Cell Host Microbe 22, 552.e5-560.e5. doi: 10.1016/j.chom.2017.09.005

Conflict of Interest: The authors declare that the research was conducted in the absence of any commercial or financial relationships that could be construed as a potential conflict of interest.

Copyright (C) 2021 Corcionivoschi, Thompson and Gundogdu. This is an open-access article distributed under the terms of the Creative Commons Attribution License (CC $B Y)$. The use, distribution or reproduction in other forums is permitted, provided the original author(s) and the copyright owner(s) are credited and that the original publication in this journal is cited, in accordance with accepted academic practice. No use, distribution or reproduction is permitted which does not comply with these terms. 